Copyright by the Acoustical Society of America. Mennitt, D. \& Johnson, M. (2010). Multiple-array passive acoustic source localization in urban environments.

Journal of the Acoustical Society of America, 127(5), 2932-2942. doi: 10.1121/1.3372743

\title{
Multiple-array passive acoustic source localization in urban environments
}

\author{
Daniel Mennitt ${ }^{\text {a) }}$ and Marty Johnson \\ Department of Mechanical Engineering, Vibration and Acoustics Laboratories, Virginia Polytechnic \\ Institute and State University, Blacksburg, Virginia 24061
}

(Received 20 December 2008; revised 20 February 2010; accepted 3 March 2010)

\begin{abstract}
In many situations of interest, obstacles to acoustic wave propagation such as terrain or buildings exist that provide unique challenges to localization. These obstacles introduce multiple propagation paths, reflections, and diffraction into the propagation. In this paper, matched field processing is proposed as an effective method of acoustic localization in a two dimensional scattering environment. Numerical techniques can be used to model complex propagation in a space where analytical solutions are not feasible. Realistically, there is always some uncertainty in model parameters that in turn can adversely affect localization ability. In particular, uncertainty in array location, sound speed, and various parameters affecting inter-array coherence only are investigated. A spatially distributed, multiarray network is shown to mitigate the effects of uncertainty. Multiarray inverse filter processing techniques are evaluated through perturbation of uncertain model parameters. These techniques are more accurate and flexible to implement than other matched field processing methods such as time reversal.
\end{abstract}

(C) 2010 Acoustical Society of America. [DOI: 10.1121/1.3372743]

PACS number(s): 43.60.Fg, 43.60.Kx, 43.60.Jn, 43.28.Js [DRD]

Pages: 2932-2942

\section{INTRODUCTION}

The ability to detect, locate, and identify acoustic sources, be they gunfire, animals, or geological events, is advantageous to a wide range of monitoring and surveillance applications. ${ }^{1}$ An approach utilizing multiple inexpensive nodes of simple acoustic sensors to form a distributed sensor network can be very effective. ${ }^{2}$ Strengths of acoustic sensors include long range, high-fidelity, no line of sight (LOS) to target, and passive nature.

Many free-field techniques are available to accomplish acoustic source localization-such as time difference of arrival, ${ }^{3}$ interaural level difference, ${ }^{4}$ and triangulation of bearings ${ }^{5}$ - which passively exploit phase and or amplitude differences between sensors. These methods are formulated assuming plane wave propagation and break down in more complicated environments such as urban areas. The major difference is the presence of terrain or buildings that act as obstacles to acoustic wave propagation. These obstacles obstruct LOS and introduce multiple propagation paths, reflections, diffraction, and scattering into the propagation. Specifically, reverberant energy distorts loudness cues and indirect paths distort phase cues and time of arrival. Localization by bearings can tolerate some cases of non-LOS, such as diffraction over a hill, but will be confused by image sources. Returns from the environment that stand prominently above the diffuse and temporally decaying reverberation background and can be confused with or camouflage returns from an intended target and are known as clutter.

The problem of multipath propagation is well known and many statistical approaches have been proposed to miti-

\footnotetext{
a) Author to whom correspondence should be addressed. Electronic mail: dmennitt@vt.edu
}

gate the effect of clutter. ${ }^{6,7}$ In a known environment, a powerful method for acoustic source localization is time reversal refocusing ${ }^{8,9}$ in which sensor measured signals are backpropagated in a virtual environment using finite-difference time-domain (FDTD) models that incorporate the geometry and propagation physics. Because of the reciprocity of the wave equation, inverted signals emitted from the sensor locations converge on the original source location. Time reversal acoustics has been developed recently in many fields, ${ }^{10,11}$ especially medical ultrasound, and focusing ability has been found to degrade when there is a loss of information. ${ }^{12}$ Specifically, dissipation in the medium breaks the invariance of wave equation and, in practice, the time reversal operation is achievable only on a limited aperture time reversal mirror ${ }^{13}$ that results in decreased focusing quality in diffracting media. Measurement of a propagation matrix, the collection of transfer functions between the time reversal mirror and multiple sources, to create an inverse filter has been shown to give superior performance to time reversal at the cost of more measurements. ${ }^{14,15}$ This relationship between time reversal and inverse filtering has been extensively studied. ${ }^{16,17}$

In this paper, matched field processing (MFP) using an inverse filter is presented as a method to locate acoustic sources in an urban environment using multiple microphone arrays. Whereas the conventional plane wave beamformer ${ }^{18}$ steers an array in a particular direction, a matched field processor exploits the full-field acoustic propagation to predict the pressure in multiple dimensions, i.e., if the field is complex enough such that it cannot be described by a single plane wave, the fields are distinct enough such that multidimension resolution is possible. The process entails matching the sensor measured acoustic field with replicas of the expected field at multiple locations, essentially an inverse problem. MFP has been largely developed in ocean 
acoustics ${ }^{19}$ for source localization and tomography. The replica fields are obtained by applying the acoustic wave equation to waveguide propagation models. In contrast to the typical underwater application, there is no closed form solution for the array manifold vectors in scattering environments due to the complex nature of propagation. The array manifold vectors can be obtained by measurement ${ }^{20,21}$ or numerical modeling. The logistic challenges of large-scale measurements compared to the widespread availability of urban geographic information and flexibility of numerical models makes this option attractive. Regardless of the employed approach, model-environment mismatch is a concern.

MFP accounts for the unique signature of the reverberant acoustic field at a receiver such that location of a propagating source can be determined anywhere in the known environment. Naturally, localization performance is correlated with the quality of a priori information. There is always some uncertainty in the precise location and even presence of scatterers, and fluctuations in the atmospheric conditions, temperature, and density of the medium are unavoidable. Mismatch has been studied extensively in the context of ocean acoustics, ${ }^{22,19}$ however, urban environments offer unique challenges. Some of the effects of model uncertainty have been studied in the context of time reversal refocusing. Liu et $a l .{ }^{23}$ found that localization was generally tolerant of minor changes in scatterer position, although the addition of an obstacle close to a receiver caused substantial degradations. Yon et al. ${ }^{15}$ reported similar robustness to perturbations such as moving persons within a room. In a second experiment, small variations in temperature were shown to increase the sidelobe level during focusing. It will be shown herein that because uncertainty in any given parameter can be interpreted as uncertainty in the phase of a propagating wave, many forms of uncertainty have similar effects.

In this paper, a network of multiple acoustic arrays is proposed to mitigate the effects of uncertain environmental parameters. One of the great strengths of sensor networks is the multitude of information from disparate vantage points. This is especially apparent in urban environments, where large obstacles can obstruct LOS and cause heavy shadowing at some receiver locations. It will be shown that the extent of error is highly dependent on the source-receiver geometry. The error induced by model mismatch is used to evaluate the relative performance of two multiarray processing methods: coherent and incoherent. While coherent processing utilizes more information, incoherent processing is more robust to distorted information. Uncertainty affecting inter-array coherence only is also investigated. Source localization with distributed sensor arrays and partial spatial coherence has been studied in the context of plane wave propagation. ${ }^{24}$ However, matched field processing using multiple arrays has not been considered previously.

In Sec. II, the signal model and signal processing methods are presented. Multiarray processing methods are introduced. It is shown how numerical modeling techniques can be used to acquire the propagation matrix for MFP. In Sec. III, an example of acoustic propagation in a complex urban environment is used to demonstrate single and multiarray processing methods. In Sec. IV, the effect of uncertainty

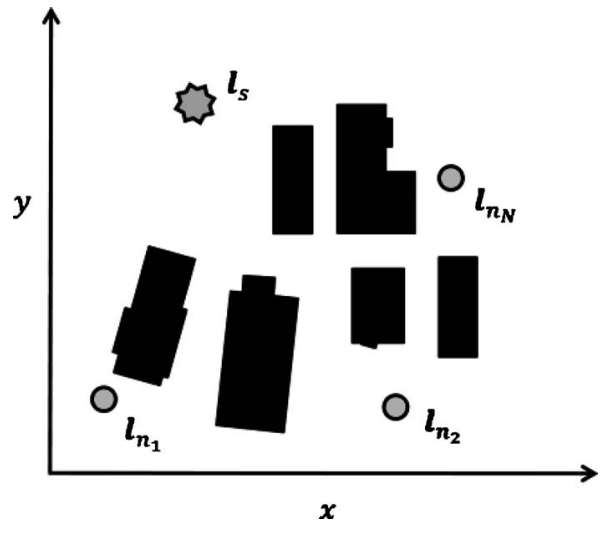

FIG. 1. Representation of an urban setting illustrating spatial sourcereceiver relationships.

(calibration error, model mismatch, and uncertainty affecting inter-array coherence only) on these methods is examined through numerical experiments to demonstrate the relative performance during practical implementation. The performance is spatially dependent and it will be shown that this technique readily lends itself to the advantages of a spatially distributed sensor network and incoherent processing.

\section{THEORY}

\section{A. Signal model}

In general, we assume small compact nodes each equipped with a microphone array, limited onboard computational ability, and a communication link to the network. Consider $N$ nodes each with $M$ sensors and a single stationary source radiating at one of $J$ discrete locations $\boldsymbol{l}_{n}$ $=\left\{x_{s}, y_{s}\right\}$. A schematic of a representative two dimensional (2D) node-source geometry appears in Fig. 1. Solid black objects represent buildings which obstruct LOS and introduce multiple propagation paths, reflections, diffraction, and scattering into the propagation.

The sampled signal at the $m$ th microphone on the $n$th node $r_{n, m, j}(t)$ consists of the $j$ th source signal $s_{j}(t)$ filtered by the environment and a noise component $v_{n, m}(t)$ as follows:

$$
r_{n, m, j}(t)=d_{n, m, j}(t) * s_{j}(t)+v_{n, m}(t),
$$

where $d_{n, m, j}(t)$ is the impulse response between source and receiver which captures the effects of propagation in a complex environment as well as the acousto-electric response of the receiver.

In the frequency domain, let $\boldsymbol{r}_{n}(\omega)$ be the vector of complex sound pressures measured by the $M$ microphones of the $n$th array and $s(\omega)$ be a vector of complex source signals radiating from $J$ locations at frequency $\omega . \boldsymbol{r}_{n}(\omega)$ and $s(\omega)$ are related by $\boldsymbol{D}_{n}(\omega)$, an $M \times J$ complex propagation matrix, where $d_{n, m, j}(\omega)$ is the transfer function between the $m$ th microphone and a sound source radiating from the $j$ th location. Therefore, we have 


$$
\begin{aligned}
& {\left[\begin{array}{c}
r_{1}(\omega) \\
\vdots \\
r_{M}(\omega)
\end{array}\right]_{n} } {\left[\begin{array}{ccc}
d_{1,1}(\omega) & \cdots & d_{1, J}(\omega) \\
\vdots & \ddots & \vdots \\
d_{M, 1}(\omega) & \cdots & d_{M, J}(\omega)
\end{array}\right]_{n}\left[\begin{array}{c}
s_{1}(\omega) \\
\vdots \\
s_{J}(\omega)
\end{array}\right] } \\
&+\left[\begin{array}{c}
v_{1}(\omega) \\
\vdots \\
v_{M}(\omega)
\end{array}\right]_{n} \\
& \boldsymbol{r}_{n}(\omega)=\boldsymbol{D}_{n}(\omega) \boldsymbol{s}(\omega)+\boldsymbol{v}_{n}(\omega) .
\end{aligned}
$$

In general, notation indicating time or frequency dependence will be suppressed when it is clear from the context.

\section{B. Propagation model}

In typical beamforming applications, free-field propagation is assumed and the propagation matrix can be described analytically by the source-receiver distance. ${ }^{18}$ Urban propagation is a very complicated problem because of the presence and often irregular structure of many intersecting streets amid variously shaped buildings. In such environments, closed form expressions for acoustic pressure between arbitrary source and receiver locations do not exist. Because of its major importance, noise propagation in urban spaces has been studied for a long time. A history of various approaches can be found by Picaut et al. ${ }^{25}$ Recent advances in computational power enable numerical models of sufficient complexity to be calculated. The FDTD method has been chosen due to its flexibility, accuracy, and ease of implementation.

The FDTD method solves a first-order approximation of the differential equations that govern acoustic wave propagation. It is possible to create very high-fidelity models which can simulate the acoustic field in the presence of complicated phenomena such as scattering obstacles, transient sources, nonlinear effects, and a moving inhomogeneous medium. ${ }^{26}$

The widely accepted staggered difference algorithm, ${ }^{27}$ or leapfrog method, is used in which velocity and pressure calculations are staggered in both time and space. A substantial reduction in computational requirements is achieved by using a two dimensional model as an approximation of the three dimensional world. This involves a number of approximations and limitations to the modeling capability; ${ }^{28}$ for example, buildings are essentially infinitely tall and vertical propagation is ignored. However, the kinematics of the computations remains accurate and the $2 \mathrm{D}$ model is sufficient to describe all phenomena of interest for the purpose of this work.

To build the propagation matrix $\boldsymbol{D}$, a FDTD simulation is run once for each source location. The pressure histories at the sensor locations are stored and a Fourier transform is performed on each impulse response to yield the frequency domain steering vectors.

\section{Matched field processing}

Matched field processing provides an estimate of the field distribution given the sample covariance matrix. The matched field power response function is the beamformer output

$$
b_{j}(\omega)=\boldsymbol{w}_{j}{ }^{H} \boldsymbol{\Phi}_{R R} \boldsymbol{w}_{j},
$$

where $\boldsymbol{w}_{j}$ is a $M \times 1$ vector of complex frequency domain sensor coefficients for the $j$ th source and $\boldsymbol{\Phi}_{R R}$ is the cross spectral density of the vector of microphone outputs. ${ }^{19}$ The real vector $\boldsymbol{b}$ is the concatenation of $j$ beamformer outputs and an estimate of the squared source location vector $s^{2}$. The source location estimate is chosen as the argument that maximizes $\boldsymbol{b}$. There are many different choices for $\boldsymbol{w}$ based on the statistics of the data received at the array. The goal is to optimize the beamformer response so the output contains minimal contributions due to noise and signals arriving from directions other than the desired signal directions.

Matched field processing is a generalization of plane wave beamforming and many algorithms developed in this context have been adapted to MFP. The minimum variance distortionless response (MVDR) beamformer has been developed in the context of plane wave beamformers ${ }^{18}$ and has been introduced to MFP, ${ }^{19}$ sometimes referred to as the maximum likelihood method. It has a number of attributes suitable to MFP; these include good sidelobe suppression and a modest tolerance to mismatch when compared to other adaptive methods. The weights are given by

$$
\boldsymbol{w}_{j}(\omega)=\frac{\boldsymbol{\Phi}_{V V}^{-1} \boldsymbol{d}_{j}}{\sqrt{\boldsymbol{d}_{j}^{H} \boldsymbol{\Phi}_{V V}^{-1} \boldsymbol{d}_{j}}},
$$

where $\mathbf{d}_{j}$ is a $M \times 1$ vector of complex frequency domain sensor coefficients for the $j$ th source. The noise-only covariance matrix $\boldsymbol{\Phi}_{V V}$ is used as it allows for flexibility in design, and inclusion of the signal in the matrix can increase sensitivity to mismatch. ${ }^{29}$

Practically, it can be difficult to obtain signal free estimates of the noise cross spectral density (CSD). Assuming a diffuse noise field creates a superdirective beamformer that optimally rejects coherent noise. However, uncorrelated noise is amplified at low frequencies where $\boldsymbol{\Phi}_{V V}$ becomes ill-conditioned. In order to overcome this problem, the MVDR beamformer can be solved under a white noise constraint. The method uses a small added scalar to the main diagonal of the CSD matrix, which also conditions the matrix for inversion.

$$
\boldsymbol{\Phi}_{V V}(\omega)=(1-\alpha) \boldsymbol{D} \boldsymbol{D}^{H}+\alpha \frac{\left\|\boldsymbol{D} \boldsymbol{D}^{H}\right\|}{M} I .
$$

The factor $\alpha$ can range from 0 to 1 , which results in the unconstrained superdirective or delay-and-sum beamformer, respectively. Here, $\alpha$ can be chosen based on the noise field and equipment used, and for this work a value of $\alpha=0.01$ is used to provide a balance between directivity index and white noise gain. This realization of $\boldsymbol{\Phi}_{V V}$ is used for all the results to follow.

\section{Multi-array processing}

Incoherent network processing implies that the individual beamformer outputs of each array are combined in a magnitude only sense; no phase information is shared. For example, the outputs could be averaged such that 


$$
b_{\mathrm{inc}}=\frac{1}{N} \sum_{n=1}^{N} \boldsymbol{b}_{n} .
$$

Any correlation that may exist between the arrays is ignored. Although the potential for this information is lost, the processor is ultimately robust against any corruption of said information.

To process the data from all arrays coherently, all the individual microphones are treated as part of a single compound array (i.e., sensor outputs $\boldsymbol{r}_{n}$ from each of the $N$ arrays are aggregated into one vector). Because the array signals are combined in a complex sense, the relative magnitude and phase information is retained. Alternatively, incoherent processing discards this information and the criterion for any given optimal weights is not met. Assuming perfect coherence between all sensors in the network, the optimal coherent method will outperform the incoherent method. However, the incoherent processor is robust to a lack of inter-array coherence. This will be explored in more detail in Sec. III.

\section{E. Broadband processing}

If the source is broadband such that there is energy distributed across some frequency bandwidth, the beamformer output can be combined across $F$ frequencies. The use of increased amounts of data stabilizes the estimation process, and has been found to be effective in reducing ambiguous beamformer sidelobes. ${ }^{30}$

There are a number of different ways to combine the beamformer outputs across frequency; these can be classified broadly into two classes: conventional incoherent methods and coherent broadband methods. ${ }^{31}$ Depending on the propagation environment, the beamformer outputs may contain useful cross frequency information if spatially correlated noise is present.

Broadband incoherent processing ignores any correlation across frequency and is optimal only in the unknown flat source spectrum case

$$
b_{B B, j}=\frac{1}{F} \sum_{f=1}^{F} \frac{b_{j}\left(\omega_{f}\right)}{\left\|\boldsymbol{b}\left(\omega_{f}\right)\right\|},
$$

where the matched field power response function $b_{j}\left(\omega_{f}\right)$ can be the result of incoherent or coherent array processing.

\section{MULTI-ARRAY MATCHED FIELD PROCESSING IN AN URBAN ENVIRONMENT}

\section{A. Test environment}

The test environment for the following numerical experiments is based on an artificial training village of 15 closely spaced concrete buildings in a flat area, similar to that used for previous time reversal experiments. ${ }^{8}$ A general consistency between the sound pressure level given by a two dimensional FDTD model and measured microphone data at multiple locations has been found in previous work by Liu and Albert. ${ }^{32}$ Given the results of the experimental validation, a similar FDTD model has been constructed for this work.

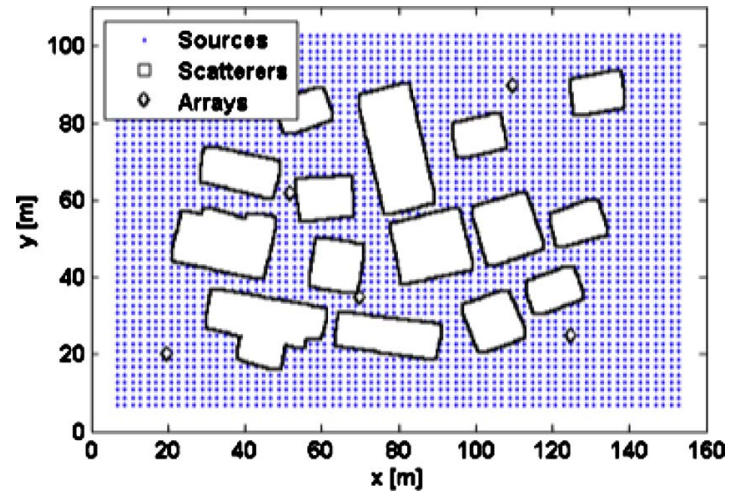

FIG. 2. (Color online) Two dimensional FDTD model of artificial training village used for the study.

Figure 2 shows the geometrical relationship between five four-microphone arrays and multiple scattering objects over a $160 \times 110 \mathrm{~m}^{2}$ area. The scattering objects are treated as rigid (infinite impedance). At the wavelengths of interest, any surface roughness present on the scattering objects is negligible. The source signal is a Ricker wavelet ${ }^{33}$ with a center frequency of $f_{c}=100 \mathrm{~Hz}$. This a zero-phase wavelet commonly used in seismology to represent impulsive sources. The FDTD spatial mesh is Cartesian with grid cells $\{\Delta x, \Delta y\}=\{0.2,0.2\} \mathrm{m}$ yielding 440000 elements overall. This allows for roughly 17 grid points per primary wavelength. Microphones are arranged in each array with radius $0.2 \mathrm{~m}$. Here, 3626 source locations are specified at $2 \mathrm{~m}$ spacing in a rectangular grid. To ensure numerical stability of the FDTD calculation, the time step is chosen to satisfy the theoretical courant condition. ${ }^{34}$ The time step is chosen as 0.3 $\mathrm{ms}$ and the simulation is run for 2500 time steps or $750 \mathrm{~ms}$ to allow the pulse excitation to sufficiently propagate through the environment. All 2500 points are used to calculate the fast Fourier transform (FFT) for subsequent frequency domain processing.

\section{B. Numerical example}

The transmission loss ${ }^{19}$ of two single arrays has been reshaped into a two dimensional matrix and laid onto the proper coordinates in relation to the scatterers (Fig. 3). In addition to spherical spreading, the sensitivity is heavily influenced by reflection, diffraction, and shadowing introduced by the scatterers. Similarly, the transmission loss of the entire coherent five-array network also appears in Fig. 3 (right pane). The transmission loss of the coherent network is distinct from any single array. Contrary to an average, it takes on the features of the most sensitive array to any given location; i.e., the coherent sensitivity is low only if the sensitivity of every individual array is.

Generally, source localization is very challenging in scattering environments due to multipath propagation. However, the MFP performance actually increases over the freefield case as complex diffraction and scattering lends spatial distinction to the acoustic field, known as hyper-resolution. ${ }^{35}$ Aubry et $a l .{ }^{36}$ has shown the complexity of a scattering medium to increase the global number of degrees of freedom of the propagation matrix. 

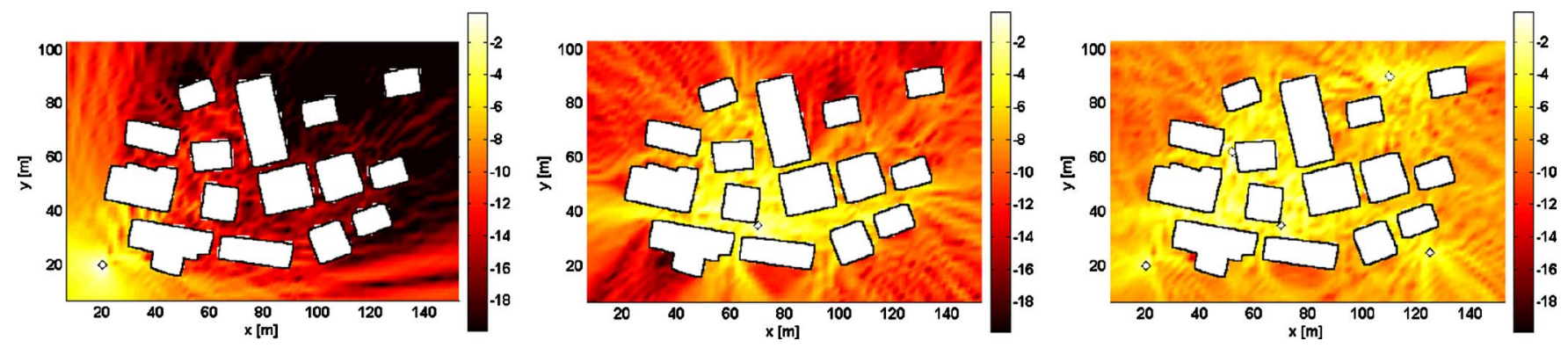

FIG. 3. (Color online) Transmission loss (dB) at $100 \mathrm{~Hz}$ of array 1 (left), array 2 (center), and the coherent five-array network (right). Primary paths for propagation through the obstacles is indicated by high transmission loss.

Considering two single arrays (node 1 and node 2) and two multiarray processing strategies (coherent and incoherent processing utilizing all five nodes), the broadband beamformer matched field power response functions corresponding to a source at $\boldsymbol{l}_{s}=\{65,73\}$ have been calculated and are presented in Fig. 4. Twenty-five discrete frequencies have been averaged in the band $90-120 \mathrm{~Hz}$, which has reduced the sidelobe level. These are also known as ambiguity functions because they show the relative uniqueness between the beamformer output at each location. In all cases, the maximum of the matched field power response function is at $\boldsymbol{l}_{s}$. Regions in space that share a similar amplitude and phase response to the true source location are essentially sidelobes as in directional beamforming. However, in MFP, the characteristics of the environment have a far greater influence on beamformer sidelobes than microphone geometry. Node 2 receives more direct energy and is in closer proximity than node 1 which receives propagation through many buildings. The complex geometry creates a distinct acoustic field; however, in a large domain, the distinction is not necessarily unique at every point as evident by sidelobes. While both the incoherent and coherent network clearly resolves the source, sidelobes are more efficiently reduced by the coherent processing. The incoherent approach is an average so while one array's contribution eliminates some of the ambiguity of another, it also adds some of its own.

\section{UNCERTAINTY AND LOCALIZATION PERFORMANCE}

In this section, some aspects of practical implementation are studied in a complex urban environment. The relative performance of individual arrays and multiarray processing techniques is evaluated considering uncertainty in model parameters. Because the effects are highly dependent on the source-receiver geometry, localization error is calculated over the entire domain. Finally, the feasibility of using freefield weights for MFP in a full-field environment is investigated.
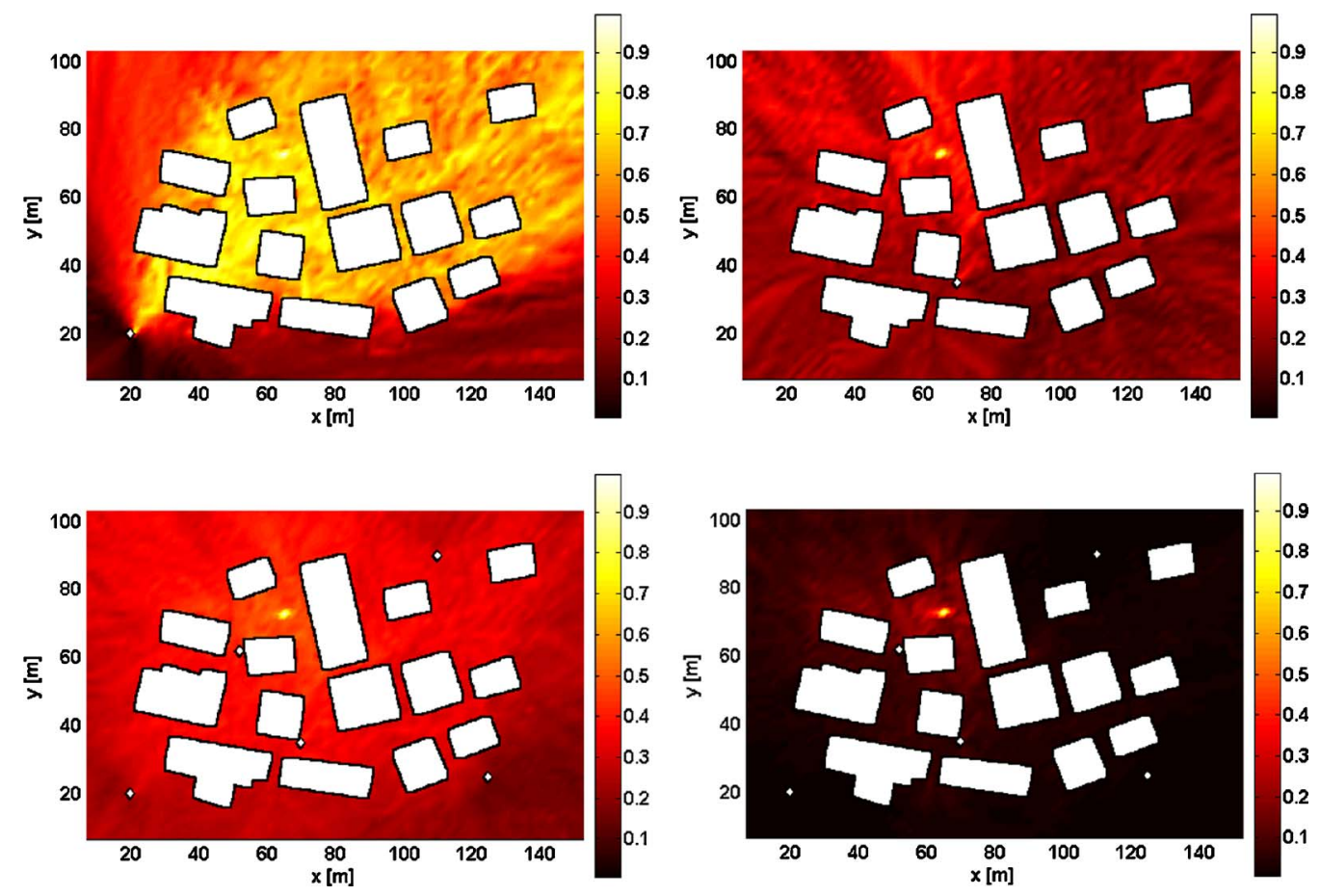

FIG. 4. (Color online) Broadband matched field power response function for array 1 (top left), array 2 (top right), the incoherent array network of five arrays (bottom left), and the coherent array network of five arrays (bottom right). The source is located at $l_{s}=\{65,73\}$. 


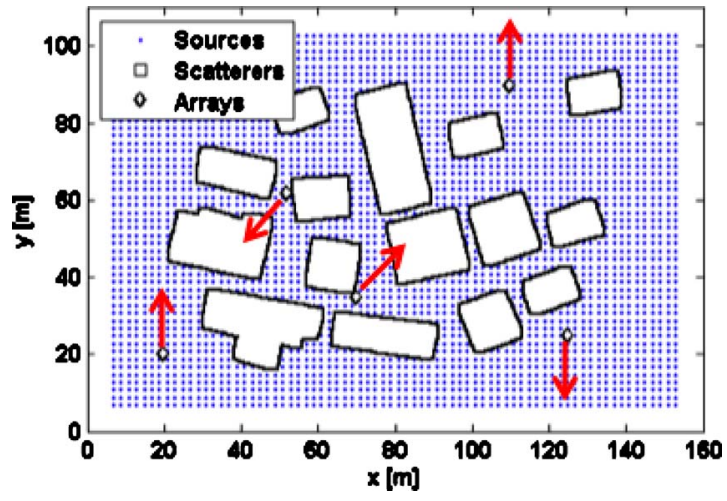

FIG. 5. (Color online) Geometry of the simulation. Arrows indicate the direction in which the node location was perturbed for the mismatch study.

\section{A. Forms of uncertainty}

The ability of the true source location to dominate the magnitude of the beamformer output will degrade in the presence of uncertainty. This uncertainty can manifest itself in a variety of ways, such as thermal noise originating from the sensors and associated hardware, noise from wind, and acoustic interferers. It is also likely that the numerical model or experimental situation used to obtain the steering vector matrix differs from the situation in which signals are measured, such as discrepancies in the geometry and impedance characteristics of buildings. Fine features at a small scale are computationally expensive to model and unlikely to be known at high levels of detail. Regardless, even an experimentally characterized array will suffer from the ever changing atmospheric conditions, temperature, and density of the propagating medium. Numerical experiments offer fine control over individual parameters. This section outlines some forms of uncertainty and how they are simulated. Each form of uncertainty is introduced in isolation with the exception of uncertainty due to inter-array coherence when explicitly noted

\section{Uncertainty due to calibration error}

It is possible that the true location of a microphone array differs from the numerical model. Even precise location measuring devices such as those utilizing global positioning system (GPS) can exhibit significant variance and drift. This is known as system mismatch or calibration error and various forms, such as array tilt, have been studied in ocean acoustics. ${ }^{19}$ It is informative to view these uncertainties in terms of the phase of a propagating or reflected wave. For example, at $100 \mathrm{~Hz}$, an uncertainty of $2 \mathrm{~m}$ represents $210^{\circ}$ of phase. Therefore, near a reflecting boundary with an uncertainty of $2 \mathrm{~m}$, we cannot be certain whether the reflected wave will be in phase or out of phase with the incoming wave at any given position. To investigate this case, a perturbed model was built in which the location of each sensor array is shifted $2 \mathrm{~m}$ in one direction (Fig. 5).

The original and perturbed node locations are listed in Table I. Data from the perturbed model simulate signals actually acquired while the data from the original model are an estimate $\hat{\boldsymbol{D}}$ of the true steering vector matrix. Because the
TABLE I. Original and perturbed array coordinates in the $x, y$ plane (meters) for the $2 \mathrm{~m}$ location uncertainty example.

\begin{tabular}{lcc}
\hline \hline & Original & Perturbed \\
\hline Array 1 & 20,20 & 20,22 \\
Array 2 & 70,35 & $71.4,36.4$ \\
Array 3 & 52,62 & $50.6,60.6$ \\
Array 4 & 110,90 & 110,92 \\
Array 5 & 125,25 & 125,23 \\
\hline \hline
\end{tabular}

relative positions of sources, sensors, and scatterers are important, results of this perturbation are indicative of reciprocal situations.

Broadband matched field power response functions for a $2 \mathrm{~m}$ uncertainty in array location are presented in Fig. 6. In general, a perturbation of the steering vectors causes the mainlobe to decrease in value while the sidelobe levels fluctuate. The precise effect of model mismatch, however, is very complex and dependent on the array-source geometry and model. Contrary to both multi-array techniques, none of the single array maps maintained the global maximum at the true source location.

In the case of mismatch, the signal model is no longer described by Eq. (2). Although the received waves do not match any cataloged steering vectors, they are coherent across the array. Uncertainty in model parameters can be interpreted as corrupting the steering vectors with spatially correlated noise. Note that, from a MFP beamformer design standpoint, this is a motivation for using the superdirective beamformer over the delay-and-sum beamformer (which optimally rejects incoherent noise).

\section{Uncertainty due to model mismatch}

Like an uncertainty in location, uncertainty in the temperature of the propagating medium can be interpreted as a phase uncertainty and has a similar effect. Consider a $20{ }^{\circ} \mathrm{C}$ perturbation in temperature. There is a $3 \%$ increase in the speed of sound between 15 and $35{ }^{\circ} \mathrm{C}$ With this change in temperature, a propagating wavefront at $100 \mathrm{~Hz}$ will be completely out of phase with a wave at the original temperature after approximately 15 wavelengths or about $52 \mathrm{~m}$. To investigate this case, a perturbed model was built in which the temperature was increased by $20^{\circ} \mathrm{C}$. Data from the perturbed model simulate signals actually acquired while the data from the original model are an estimate $\hat{\mathbf{D}}$ of the true steering vector matrix. Visual results for this case are suppressed for brevity, but will be addressed in Sec. IV B.

\section{Uncertainty affecting inter-array coherence only}

Atmospheric turbulence and uncertainty in parameters such as the speed of a moving medium are likely to have a negligible effect on the closely spaced sensors of a single array but can cause substantial phase differences on a larger scale between microphones on separate nodes. ${ }^{37}$ Furthermore, data from each node are referenced to an individual clock, which is subject to desynchronization with the network. All of these factors compromise inter-array signal coherence. 

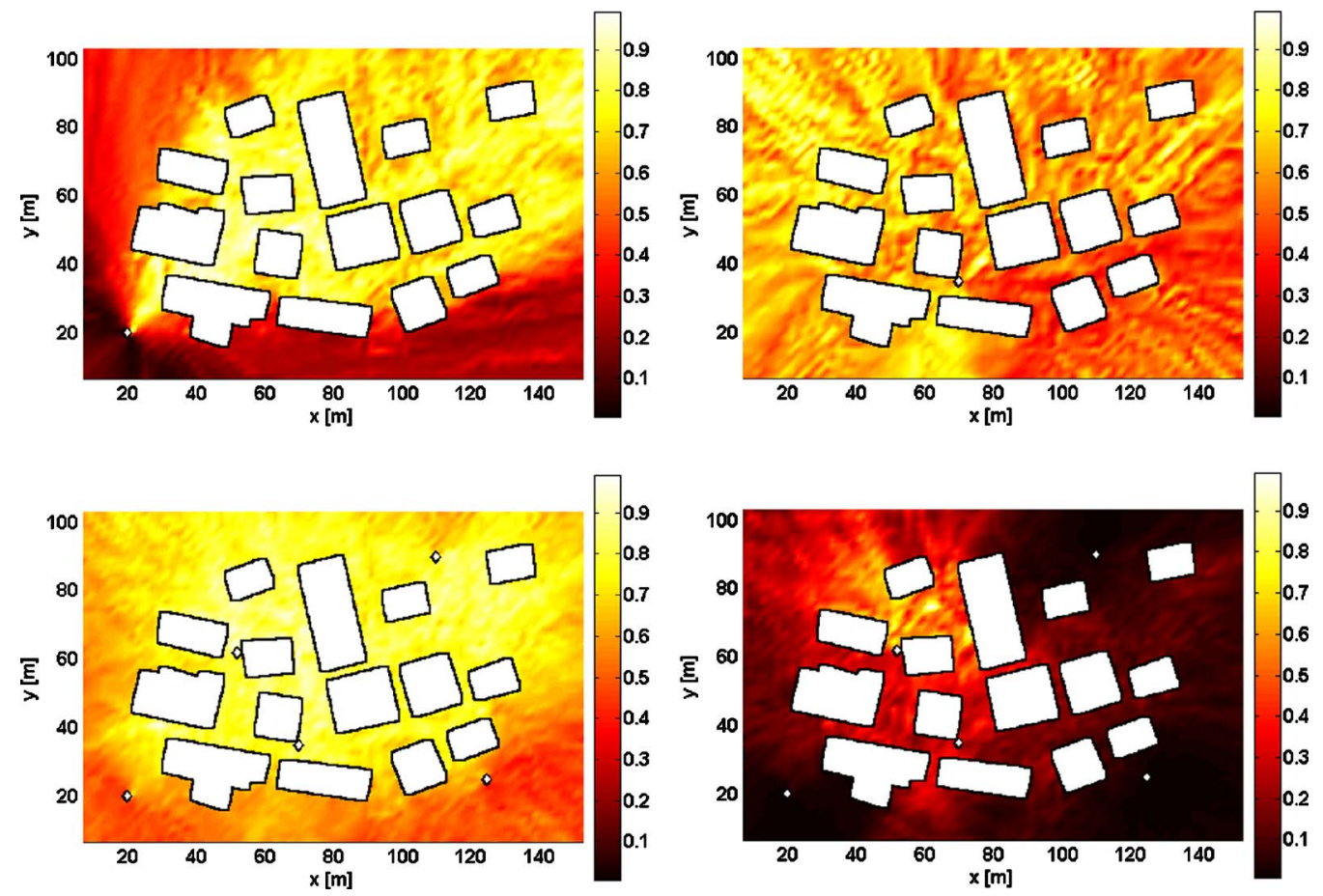

FIG. 6. (Color online) Broadband matched field power response function with a $2 \mathrm{~m}$ uncertainty in node location for node 1 (top left), node 2 (top right), the incoherent network of five arrays (bottom left), and the coherent network of five arrays (bottom right). The source is located at $l_{s}=\{65,73\}$.

To simulate perfect intra-array coherence while degrading inter-array coherence, each array's signal vector $\mathbf{r}_{n}$ is phase shifted by a realization of a random phase factor

$$
\zeta=e^{i \pi \beta v},
$$

where $i=\sqrt{-1}, v \in\{0,1\}$ is a uniformly distributed random variable and $\beta \in\{0,2\}$ is a parameter governing the extent of phase distortion. Over many averages, the spectral coherence function matrix of the compound array becomes block diagonal, with ones on the block diagonal (corresponding to individual arrays) and reduced inter-array coherence elsewhere. Here, we confine our study to the limiting case of zero inter-array coherence induced by $\beta=2$. Although a range of coherence is possible, this example represents the worst-case scenario. Despite precautions such as using the best available hardware, in practical implementation it is often unrealistic to assume a level of coherence due to the variability of the propagation medium (turbulence, wind, temperature gradients, etc.).

Figure 7 displays two cases of a broadband coherent network matched field power response function due to an absence of inter-array coherence. More robust than timedomain methods, the frequency domain formulation decouples the magnitude and phase and coherent processing is still viable even though coherence between arrays is 0 . The first case, Fig. 7 (top), considers the effect of zero inter-array coherence only. Compared to Fig. 4, the result is a very similar response to the unperturbed coherent case with the addition of increased sidelobes due to the corrupted phase information. However, the sidelobes are not as high as the incoherent processor which does not utilize relative amplitude information. In contrast to typical free-field beamforming, the relative amplitude provides discriminating informa-
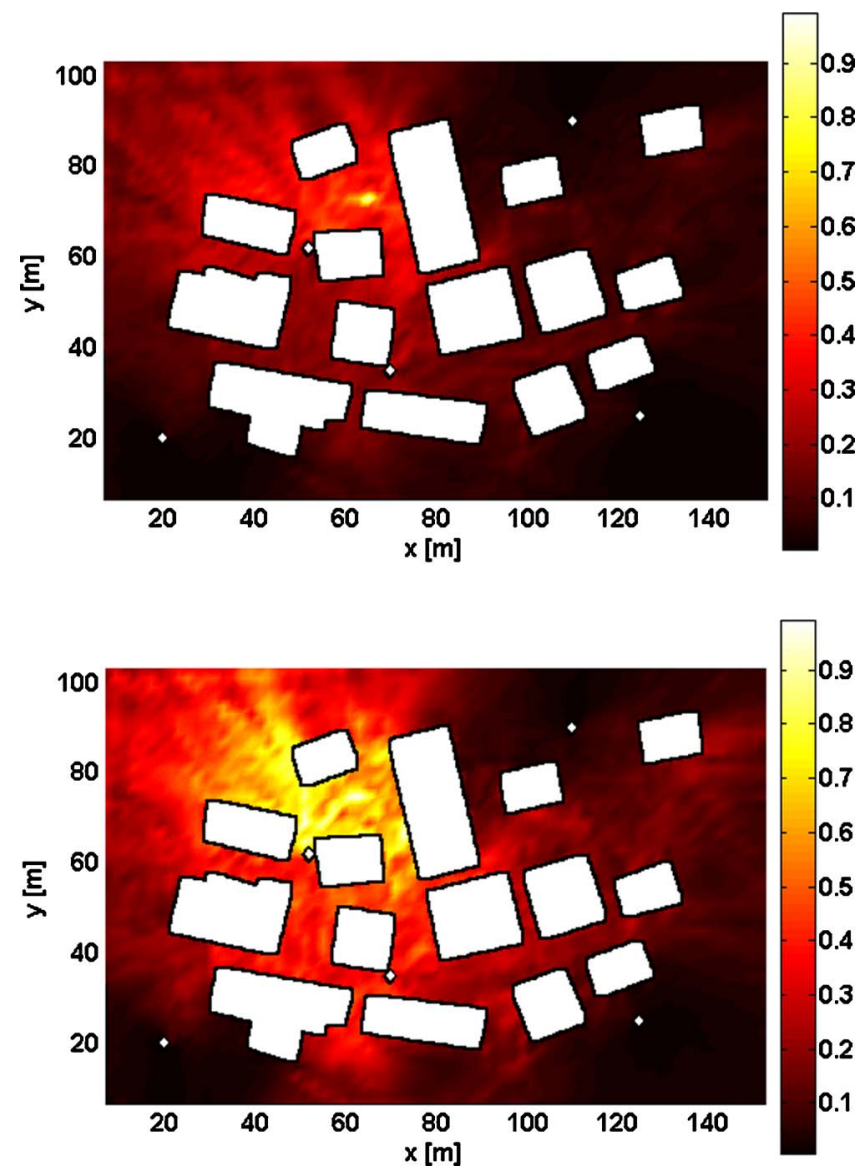

FIG. 7. (Color online) Broadband matched field power response function for the coherent network of five arrays with zero inter-array coherence to be compared with Fig. 4 (no mismatch, top) and Fig. 6 (2 m location perturbation, bottom). 
tion useful in matched field processing. Figure 7 (bottom) is the coherent network response subject to both a $2 \mathrm{~m}$ perturbation in phase and absence of inter-array coherence. Compared to Fig. 6 (bottom right), again we see increased sidelobes and a peak that has shifted farther from the correct location. In this case, both the relative phase and amplitude information is distorted. A loss of inter-array coherence alone is not enough to disrupt localization; it intensifies the effect of other uncertainty

\section{B. Spatial variation of performance}

While many studies have been done on the consequences of imperfect models ${ }^{23}$ and it is almost always assured that model uncertainty will degrade beamformer performance, the extent of the degradation is both model specific and highly dependent on the array-source geometry as shown previously. To quantify the impact of mismatch on performance, it is necessary to search a large domain. In the past, this has been difficult as many time-consuming computations must be carried out. With the availability of ever increasing computational power, however, simulations of useful scope can be executed reasonably. The localization performance of each individual array and the entire network considering various forms of uncertainty was calculated over the entire domain.

The rms localization error $\varepsilon_{j}$ of the estimated source location versus the true source location is calculated as

$$
\varepsilon_{j}=\sqrt{\left(\hat{\boldsymbol{l}}_{j}-\boldsymbol{l}_{j}\right)^{T}\left(\hat{\boldsymbol{l}}_{j}-\boldsymbol{l}_{j}\right)},
$$

where $\hat{l}_{j}$ is chosen as the location that maximizes the broadband matched field power response function $\boldsymbol{b}_{B B, j}$. The spatial distribution of error considering a $2 \mathrm{~m}$ uncertainty in array location appears in Fig. 8 for individual arrays and the various network processing schemes.

Turning attention first to the individual array performance, there is a trend of increasing error with distance, especially visible for arrays outside the cluster of buildings. Possibly, it is more accurate to say the error increases with each interaction with a reflecting boundary where the phase mismatch causes constructive versus destructive interference or vice versa. Uncertainty in temperature (not shown) yields similar trends.

Although individual arrays may perform well locally, clearly in this environment a network of spatially distributed sensors offers a dramatic increase in performance overall. The coherent processor preserves the effective range of each array and error builds up mostly on the edges. This trend is further pronounced in the coherent processor with additional inter-array incoherence. Meanwhile, the incoherent processor can be susceptible to the influence of one heavily degraded array. However, it is still able to utilize the multiarray information with some degree of effectiveness, on average outperforming any single array. While the maximum value of a single array's beamformer output may be way off mark, the incoherent processor accounts for any energy that is present at the correct location.

Statistics of the performance considering other forms of uncertainty are collected in Table II. In practical situations, an estimate that is wrong by $50 \mathrm{~m}$ and an estimate that is wrong by $100 \mathrm{~m}$ may be equally worthless. The median, as well as the mean of the localization error, is included as the median does not share the mean's susceptibility to influence by outliers.

The incoherent processor actually outperforms the coherent processor (with perfect inter-array coherence) in the perturbed temperature case. Because the coherent processor depends on more information than the incoherent, it is more vulnerable to mismatch. When inter-array coherence is completely eliminated (labeled coherent with $\zeta$ ), the coherent processor degrades past the incoherent approach. The coherent processor may have less of a margin over the incoherent processor in the temperature case because the location perturbation affected all the sensors on each array similarly. Considering the improbability of coherent conditions, incoherent processing is a robust alternative with reasonable accuracy.

For each source location, the error of the best and worstperforming individual array was recorded. Statistics for these two virtual arrays (which could be array 1, 2, 3, 4, or 5 depending on the situation) were calculated and appear in the last two rows of Table II. It is interesting to see that, at the majority of locations, a single array is more accurate than the entire network. This suggests that there may be a more favorable way to fuse array information.

\section{Parametric performance study}

The susceptibility of MFP to model mismatch raises the following question: Do conditions exist in which it is advantageous to abandon the full-field model and rely on a freefield model? Free-field techniques are attractive because they do not require a large computational or knowledge investment. Furthermore, simplicity often goes hand in hand with robustness. The free-field weights are susceptible to temperature changes, for example, but not to the degree a numerical model is. Moreover, they are completely independent of any information concerning the location of buildings. A free-field beamformer will indicate the direction from which energy originated and, in the limit of having infinite arrays, good performance is assured. Practically, the quantity of sensors is limited. In this subsection, the rate of change of performance with the number of available nodes is investigated. This study is carried across multiple scenarios: MFP using 2D free-field weights and using full-field weights under variable degrees of uncertainty.

The various cases are evaluated by the metric of percent coverage. Previous results concentrated on the absolute deviation of the location estimate. Practically, at some point, the magnitude of error is irrelevant; i.e., the estimate may be useless whether it is 60 or $600 \mathrm{~m}$ away. Percent coverage is defined here as the percentage of total locations $J$ at which the error $\varepsilon_{j}$ is less than some threshold. Figure 9 shows the percent coverage for five situations using a threshold of 20 m: MFP using free-field weights, MFP using full-field weights considering a perturbation in array location of 1,2 , 5 , and $9 \mathrm{~m}$. Each array is perturbed progressively farther in one direction as indicated by Fig. 5. Both the free-field and 

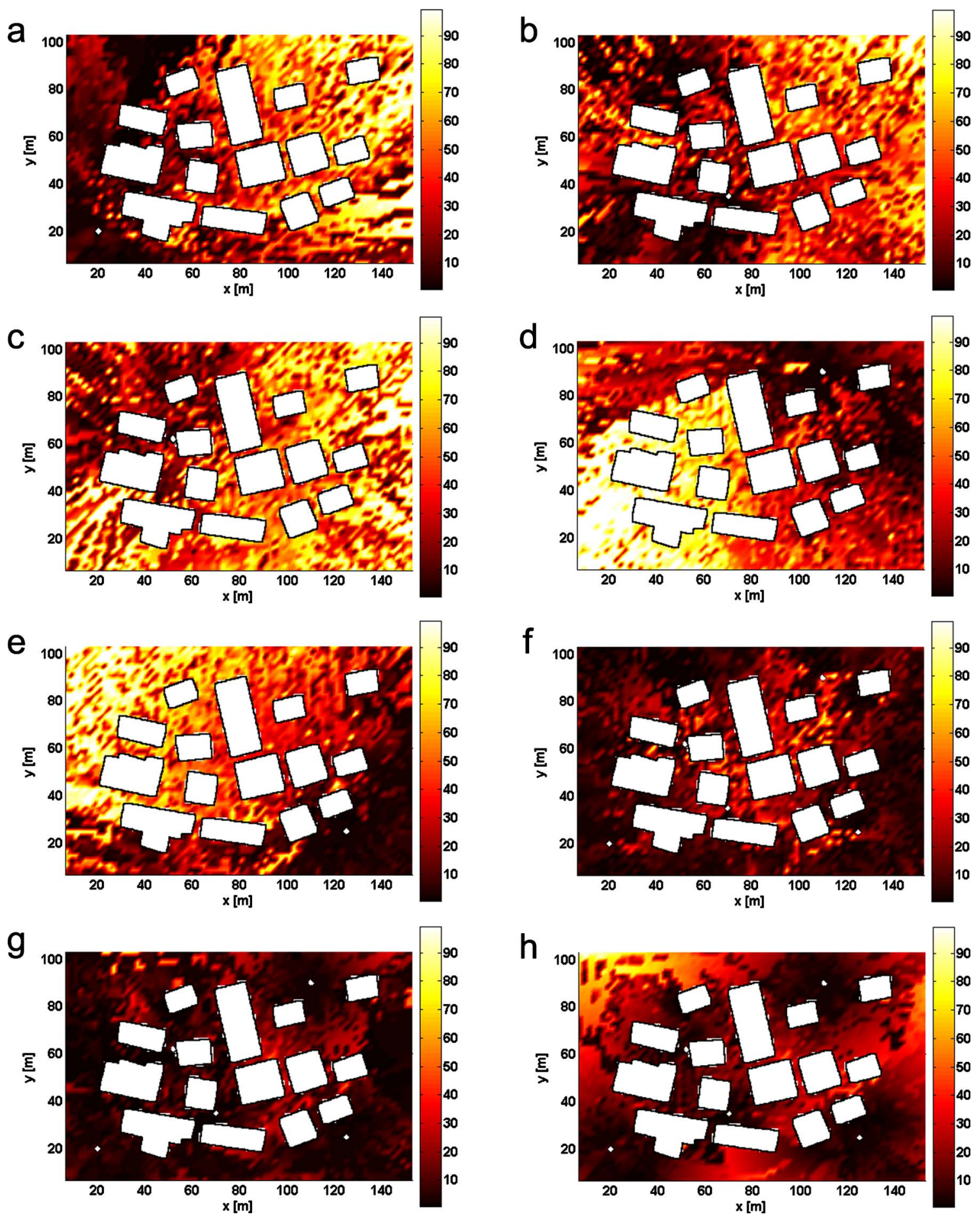

FIG. 8. (Color online) Error (meters) of matched field processor due to a $2 \mathrm{~m}$ uncertainty in array location for individual arrays [(a)-(e)], incoherent processor (f), coherent processor ( $\mathrm{g}$ ), and coherent processor with additional inter-array incoherence (h).

full-field situations utilize incoherent processing with one, two, three, four, or five nodes. For each perturbation, the results shown for the one node case is the average percent coverage of the five individual nodes. For the two node case, the performance of all possible pairs of nodes is averaged. Similarly, the three and four node case is an average of all possible triplets and quadruplets. The value for the five node case represents a single number.

As presented explicitly early in this paper, the effect of uncertainty is very complex over space. The results in Fig. 9 consider only one spatial distribution of arrays in one arrangement and density of buildings and location perturbation along a single direction. Despite the noted limitations, the performance is evaluated at a reasonable sample size of over 3000 locations. It should be noted that the free-field results for the single array represent a consequence of the simulation more than physical performance. Although on some numerical scale there are slight inter-microphone phase differences, passive ranging with a single, small aperture array is ambiguous. 
TABLE II. Mean and median error (meters) for various processing approaches under the influence of uncertainty in various parameters: medium temperature $\left(20^{\circ}\right.$ perturbation) and array location ( $2 \mathrm{~m}$ perturbation).

\begin{tabular}{lccccc}
\hline \hline & \multicolumn{2}{c}{ Location uncertainty } & & \multirow{2}{*}{ Temperature uncertainty } \\
\cline { 2 - 3 } \cline { 5 - 6 } & Mean & Median & & Mean & Median \\
\hline Array 1 & 30.2 & 17.9 & & 30.5 & 16.5 \\
Array 2 & 27.2 & 20.4 & & 24.7 & 20 \\
Array 3 & 40.1 & 32.1 & & 35.2 & 30.5 \\
Array 4 & 36.1 & 24.1 & & 27.4 & 17.1 \\
Array 5 & 31.4 & 20.9 & & 28 & 16.5 \\
Incoherent & 10.3 & 5.7 & & 8.1 & 4.5 \\
Coherent & 7.1 & 2.8 & & 8.7 & 4.5 \\
Coherent with & 15.6 & 10.2 & & 15.7 & 10.2 \\
Best array & 5.2 & 2 & & 5.8 & 4 \\
Worst array & 69.9 & 75.9 & & 62.1 & 65.3 \\
\hline \hline
\end{tabular}

Two intuitive trends are confirmed by Fig. 9: Performance decreases as the level of uncertainty increases and performance increases as the number of nodes (and therefore available information) increases. Furthermore, it is advantageous to use mildly uncertain model data over a free-field model for MFP weights, at least in a test environment of this complexity. Based on typical GPS equipment/geospatial data, uncertainty in the geometry of the boundaries (position and size of buildings and land contours) is approximately 1 $\mathrm{m}$. The movement of vehicles in an urban environment can be interpreted as an additional uncertainty of geometry on the order of $0-5 \mathrm{~m}$, depending on the proximity of the obstacle to the array. ${ }^{23}$

\section{CONCLUSIONS}

On a coarse scale, acoustic propagation in an urban environment is analogous to the reverberant field created by scattering objects. The complex field gives rise to a unique relationship between any two points in space that can be exploited for source localization. Matched field processing combined with finite-difference time-domain modeling has been proposed as an effective method of acoustic localization in a scattering environment.

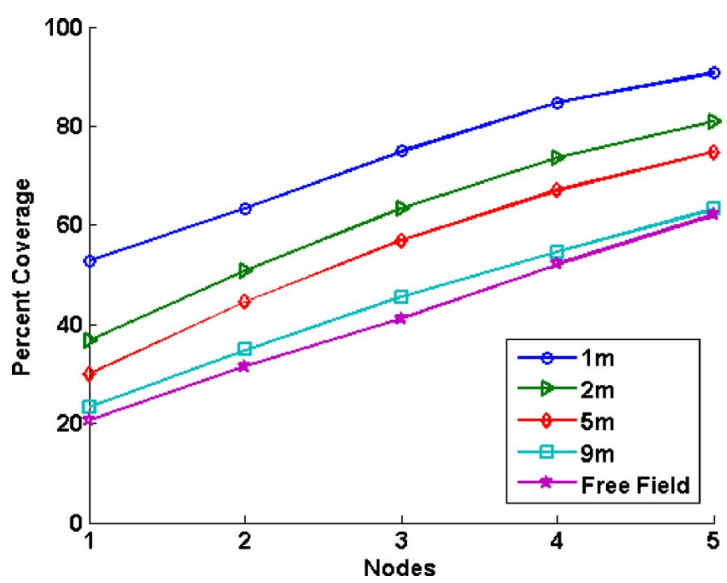

FIG. 9. (Color online) Percent coverage (error $<20 \mathrm{~m}$ ) of full-field weighted MFP considering increasing levels of uncertainty and free-field weighted MFP.
Realistically, there is always some uncertainty in various model parameters that in turn can adversely affect localization ability. Uncertainty in physical parameters can lead to uncertainty in the magnitude and/or phase of the measured signal. Phase uncertainty can also be interpreted as a loss of coherence between sensors and/or arrays. The uncertainty manifests itself as error in the position estimate and some insight into the mechanism was garnered by quantifying the error over space. It was found that the degree of performance degradation is dependent on the spatial source-receiver relationship.

A spatially distributed, multiarray network was proposed and shown to mitigate the effects of uncertainty. Processing techniques were evaluated through perturbation of uncertain model parameters. Because individual arrays are spatially separated and subject to clocking errors and large-scale medium inhomogeneities, inter-array coherency is compromised. Coherent as well as incoherent fusion strategies were studied. While the coherent processor is able to make use of more information, it is ultimately more vulnerable to mismatch. Furthermore, coherent processing generally requires higher computational and communication requirements, a luxury for untethered nodes. Results show the spatially averaged performance of coherent processing can be worse than the incoherent processor. However, the ability to utilize more information is not always detrimental if applied robustly; e.g., incoherent multiarray inverse filtering uses more information than time reversal refocusing. In a related experiment, the time reversal refocusing technique has been applied to the scenario considered herein and shown to yield inferior localization performance compared to the coherent inverse filter. ${ }^{38}$ While it is only certain that each array is locally accurate, one individual array could be identified to outperform the multiarray strategies over the majority of the domain. This suggests that there may be a more favorable way to fuse array information, and the future understanding of factors that influence array performance in such complex situations will yield superior array positioning and fusion strategies.

Use of a full-field model was shown to be more accurate than a free-field model considering modest uncertainty. It is possible that in real propagation conditions, the margin between free-field weights and numerical model based weights may be narrower. Semi-porous obstacles with finite impedance and three dimensional propagation can facilitate LOS while the increase in complexity heightens the potential for mismatch in the numerical model.

Regardless of the uncertainty level, multiarray MFP by way of an inverse filter is an attractive alternative to time reversal refocusing for passive acoustic source localization in an urban environment. In addition to superior performance, inverse filtering offers flexibility of implementation and control over design parameters through an established beamforming framework. Because beamforming can be formulated in the frequency domain, it is not necessary to transmit the full time-domain data and time synchronization requirements can be relaxed for incoherent processing. Sensor data can be locally processed at each array and only the distilled information need be transmitted to be fused with other ar- 
rays. The time accuracy required is on the order of an acoustic event as opposed to a sample. A disadvantage of this inverse method is that many calculations are needed to build the propagation matrix, even for conservative resolution of the source location vector. However, these calculations can be made before implementation and stored for real-time source localization.

${ }^{1}$ C.-Y. Chong, S. P. Kumar, and B. A. Hamilton, "Sensor networks: Evolution, opportunities, and challenges," Proc. IEEE 91, 1247-1256 (2003).

${ }^{2}$ D. Mennitt, P. Gillett, J. Carneal, and M. Johnson, "Tracking noise sources using multiple mobile microphone arrays," Proceedings of the ICSV13 (2006), pp. 171-178.

${ }^{3}$ S. P. Drake and K. Dogancay, "Geolocation by time difference of arrival using hyperbolic asymptotes," Proceedings of the ICASSP (2004), pp 361-364.

${ }^{4}$ S. T. Birchfield and R. Gangishetty, "Acoustic localization by interaural level difference," Proceedings of the ICASSP (2005), pp. 1109-1112.

${ }^{5}$ L. M. Kaplan, Q. Le, and P. Molnár, "Maximum likelihood methods for bearings-only target localization," Proceedings of the ICASSP (2001), pp. 3001-3004.

${ }^{6} \mathrm{D}$. Carevic, "Tracking target in cluttered environment using multilateral time-delay measurements," J. Acoust. Soc. Am. 115, 1198-1206 (2004).

${ }^{7}$ M. K. Broadhead, L. A. Pflug, and R. L. Field, "Use of higher order statistics in source signature estimation," J. Acoust. Soc. Am. 107, 25762585 (2000).

${ }^{8}$ D. G. Albert, L. Liu, and M. L. Moran, "Time reversal processing for source location in an urban environment," J. Acoust. Soc. Am. 118, 616619 (2005)

${ }^{9}$ D. R. Jackson and D. R. Dowling, "Phase conjugation in underwater acoustics," J. Acoust. Soc. Am. 89, 171-181 (1991).

${ }^{10}$ M. Tanter, J.-F. Aubry, J. Gerber, J.-L. Thomas, and M. Fink, "Optimal focusing by spatio-temporal inverse filter. I. Basic principles," J. Acoust. Soc. Am. 110, 37-47 (2001)

${ }^{11}$ K. G. Sabra, P. Roux, H. C. Song, W. S. Hodgkiss, W. Kuperman, T. Akal, and M. Stevenson, "Experimental demonstration of iterative time-reversed reverberation focusing in a rough waveguide: Application to target detection," J. Acoust. Soc. Am. 120, 1305-1314 (2006).

${ }^{12}$ J.-L. Thomas and M. A. Fink, "Ultrasonic beam focusing through tissue inhomogeneities with a time reversal mirror: Application to transskull therapy," IEEE Trans. Ultrason. Ferroelectr. Freq. Control 43, 1122-1129 (1996).

${ }^{13}$ D. Cassereau and M. Fink, "Time reversal of ultrasonic fields. III. Theory of the closed time reversal cavity," IEEE Trans. Ultrason. Ferroelectr. Freq. Control 39, 579-592 (1992).

${ }^{14}$ M. Tanter, J.-L. Thomas, and M. Fink, "Time-reversal and the inverse filter," J. Acoust. Soc. Am. 108, 223-234 (2000).

${ }^{15}$ S. Yon, M. Tanter, and M. Fink, "Sound focusing in rooms. II. The spatiotemporal inverse filter," J. Acoust. Soc. Am. 114, 3044-3052 (2003).

${ }^{16} \mathrm{~A}$. Tarantola, "Theoretical background for the inversion of seismic waveforms, including elasticity and attenuation," Pure Appl. Geophys. 128, 365-399 (1988).

${ }^{17}$ C. Prada and J.-L. Thomas, "Experimental sub-wavelength localization of scatterers by decomposition of the time reversal operator interpreted as a covariance matrix," J. Acoust. Soc. Am. 114, 235-243 (2003).

${ }^{18}$ J. Bitzer and K. Simmer, "Superdirective microphone arrays," Microphone
Arrays: Signal Processing Techniques and Applications (Springer, New York, 2001), pp. 19-38.

${ }^{19}$ A. B. Baggeroer, W. A. Kuperman, and P. N. Mikhalevsky, "An overview of matched field methods in ocean acoustics," IEEE J. Ocean. Eng. 18, 401-424 (1993).

${ }^{20}$ J. Carneal, M. Johnson, and P. Gillett, "Comparison of a diffracting and a non-diffracting circular acoustic array," Proceedings of the ICASSP (2006), pp. 1081-1084

${ }^{21}$ L. Fialkowski, M. Collins, W. Kuperman, J. Perkins, L. Kelly, A. Larsson, J. Fawcett, and L. Hall, "Matched-field processing using measured replica fields," J. Acoust. Soc. Am. 107, 739-746 (2000).

${ }^{22}$ D. F. Gingras, "Methods of predicting sensitivity of matched-field processors to mismatch," J. Acoust. Soc. Am. 86, 1940-1949 (1989).

${ }^{23}$ L. Liu, D. G. Albert, and D. K. Wilson, "The effect of changing scatterer positions on acoustic time-reversal refocusing in a 2D urban environment at low frequencies," J. Geophys. Eng. 4, 276-284 (2007).

${ }^{24}$ R. J. Kozick and B. M. Sadler, "Source localization with distributed sensor arrays and partial spatial coherence," IEEE Trans. Signal Process. 52, 601-616 (2004).

${ }^{25}$ J. Picaut, J. Hardy, and L. Simon, "Sound propagation in urban areas: A periodic disposition of buildings," Phys. Rev. E 60, 4851-4859 (1999).

${ }^{26}$ V. E. Ostashev, D. K. Wilson, L. Liu, D. F. Aldridge, N. P. Symons, and D. Marlin, "Equations for finite-difference, time-domain simulation of sound propagation in moving inhomogeneous media and numerical implementation," J. Acoust. Soc. Am. 117, 503-517 (2005).

${ }^{27} \mathrm{~K}$. Yee, "Numerical solution of initial boundary value problems involving Maxwell's equations in isotropic media," IEEE Trans. Antennas Propag. 14, 302-307 (1966).

${ }^{28}$ L. Liu and D. G. Albert, "Acoustic pulse propagation near a right-angle wall,” J. Acoust. Soc. Am. 119, 2073-2083 (2006).

${ }^{29} \mathrm{H}$. Cox, "Resolving power and sensitivity to mismatch of optimum array processors," J. Acoust. Soc. Am. 54, 771-785 (1973).

${ }^{30}$ A. B. Baggeroer, W. A. Kuperman, and H. Schmidt, "Matched field processing: Source localization in correlated noise as an optimum parameter estimation problem," J. Acoust. Soc. Am. 83, 571-587 (1988).

${ }^{31}$ C. Soares and S. M. Jesus, "Broadband matched-field processing: Coherent vs incoherent approaches," J. Acoust. Soc. Am. 113, 2587-2598 (2003).

${ }^{32}$ L. Liu and D. G. Albert, "Sound propagation in an urban environment. II: Preliminary FDTD model (A)," J. Acoust. Soc. Am. 114, 2442 (2003).

${ }^{33}$ R. E. Sheriff, Encyclopedic Dictionary of Exploration Geophysics, 3rd ed. (Society of Exploration Geophysicists, Tulsa, OK, 1994), Vol. 376.

${ }^{34}$ L. R. Lines, R. Slawinski, and R. P. Bording, "A recipe for stability of finite-difference wave-equation computations," Geophysics 64, 967-969 (1999).

${ }^{35}$ A. Derode, A. Tourin, and M. Fink, "Time reversal in multiply scattering media," Ultrasonics 36, 443-447 (1998).

${ }^{36}$ J.-F. Aubry, M. Tanter, J. Gerber, J.-L. Thomas, and M. Fink, "Optimal focusing by spatio-temporal inverse filter. II. Experiments. Application to focusing through absorbing and reverberating media," J. Acoust. Soc. Am. 110, 48-58 (2001).

${ }^{37}$ D. K. Wilson, "Performance bounds for acoustic direction-of-arrival arrays operating in atmospheric turbulence," J. Acoust. Soc. Am. 103, 13061319 (1998).

${ }^{38}$ D. Mennitt, "Multiarray passive acoustic localization and tracking," Ph.D. thesis, Virginia Polytechnic Institute and State University, Blacksburg, VA, 2008. 